\title{
The Importance of Review Articles \& its Prospects in Scholarly Literature
}

\begin{abstract}
Adam A Bahishti
AIJR Publisher

Corresponding author email: adam@aijr.org

Received: 12 September 2021 / Published: 12 September 2021

ABSTRACT

With the growing number of scholarly publications around the world, it is becoming more and more difficult to keep up to date with all of it. To address this issue, authors often write an insightful review article on the recent developments within their research field based on the critical review of already published original research articles. Review articles are papers that compile, summarize, critique, and synthesize the available research information on a topic. Reviews can serve as a basis for knowledge development, create guidelines for policy and practice, provide evidence of an application, highlight research gap, and if well conducted, have the capacity to engender new ideas and serve as the grounds for future research directions. To be concise, extensive review articles play an important role as a foundation for all types of research \& its application. In this editorial, I will highlight the importance of review articles along with expectations from the author who intends to submit their insightful reviews to this journal "Extensive Reviews". I encourage research scholars to write insightful and extensive review articles as a service to the scientific community and to enhance their own knowledge.
\end{abstract}

Keywords: Review Articles, Scholarly Literature, Importance of Reviews.

\section{Why Should You Write Review Articles?}

Every original research publication offers newer ideas and perspectives, research outcomes, insights into methodology, and refined understanding [1]. With the huge amount of information produced daily all around the world, identifying relevant, up to date evidence and impact has become a challenge. Additionally, for researchers new to the field, it can be hard to get an overview about a research area, for example what has been studied and general trends over time. We therefore stop and ask ourselves, "Where do we stand?" and "What do we still need to explore?" The obvious need for such curiosity sows the seeds for scholarly reviews that crystallize the gather research achievements on a particular topic in an updated, comprehensive, unbiased and holistic manner. This increases both the importance and the demand of review articles that are typically based on already published research work and is meant to summarize the available research studies about a focused topic in an organized manner. Reviewing the published research provide a mean for discussion of controversies and inconsistencies in prior research, evaluate existing methodological approaches or possibly propose future research direction. Some review articles adopt a more systematic estimation approach, whereas others are more narrative that allow the readers to get a landscape view of a topic.

Review articles are important and worth writing as a good review paper can represent the state of knowledge, explain apparent contradictions, identify required research, and even create a consensus where none existed before [2]. Writing a good review is a service to the scientific community that can also help to advance your career. Well written review articles are often highly cited and could increase the visibility and reputation of the authors. They demonstrate that you are a good synthetic thinker having mastered a research topic that may be important to your organization and hence helpful in recognition and promotion. 
Finally, if you are in a situation of not producing original research work for any reason, writing a review allows you to continue adding to your publication record.

\section{Introducing Extensive Reviews Journal}

AIJR is introducing a specific journal "Extensive Reviews" to publish exclusively review articles from all disciplines. Extensive Reviews (Extsv Rev) is a peer-reviewed, international journal where each review article will undergo standard reviewing process [3] before acceptance for publication. This journal will serve as a true multidisciplinary review journal with subject categories and subcategories, which will enable better accessibility for readers to filter reviews relevant to their research interest. During the article submission, author may select most appropriate subject category and subcategory as per review topic. If the review article represents interdisciplinary research topic, the author may select multiple categories \& subcategories for better accessibility to specific readers of interest.

To submit an insightful review article in this journal, author shall prepare well-structured review article that shall include major elements of a review article such that; Title, Authors with affiliation, Abstract, Keywords, Introduction, Main Body with Suitable Headings, Discussion, Conclusion, Competing Interests, References, etc. with at least 50\% recent reference citations. While submitting review article to Extensive Reviews, author shall avoid simultaneous submission as we take such author misconduct very seriously [4].

\section{Who Should Write Review Articles?}

A review article is a kind of research on research; or more precisely, a scholarly material with the aim to discuss already published research information about a specific topic. Writing a review article is a great responsibility as it's expected to be highly reliable, especially concerning its sources and findings. Therefore, to write a review article, it is compulsory that you are a real expert in the field of study. Compared to other article types, reviews involve fewer authors, who are usually the experts in their fields. Preparing a scholarly review might be an academically gratifying project but it can often be an arduous task. Not only is it time consuming, but also unrestricted access to metadata and full texts through electronic databases is obligatory for systematic literature search and proper interpretation of data.

Many reviews are written by researchers who study the topic being reviewed. They are in a good position to review the topic; but unfortunately, they also are biased in favor of their own results and against those who have contradicted them. The ideal review writers can be the users of the research data rather than generators of research data on a particular topic as they are likely to have a practical view of the issues and to be less biased. Workshop participants may also be the authors of unbiased reviews because their diverse points of view can increase the likelihood of identifying all relevant publications, and promote critical interpretations. As a researcher, writing a review article can be very beneficial to your career, and set you apart as an expert in your field of interest. But it also can be an overwhelming task of searching, assessing and discussing the research work in a perspective and organized manner, so that it fits the structural pattern of a literature review. Nevertheless, your perfectly written review article may impact many global researchers.

To ensure the appropriateness of authorship, its mandatory to provide the highest academic degree and/or current working or studying status for each author while submitting review article to Extensive Reviews Journal.

\subsection{Reducing Bias in a Review Article}

It is important to avoid biased reviews whether intentional or not, that can affect all stages of writing a review article including literature sources identification, research articles selection and your evaluation of the evidence. Using a protocol with well-defined inclusion or exclusion criteria can be a useful approach to reduce bias in writing a review article. Begin by determining the objectives of your review that will help to set boundaries and focused keywords selection as well as defining sections that address specific areas or research questions. Next identify multiple and unrestricted sources for your reference material to obtain a 
more comprehensive collection of recent research information. Finally, bias can also be introduced by your own interpretation of published research data and hence critical analysis of all selected research work is necessary.

To address possible bias issue, Extensive Reviews discourage excessive self-citation that should be less than $20 \%$ of total citations.

\section{How to Write a Review Article?}

The most important decision in developing a review article is selecting an appropriate topic. In general, the topic should be something in which you and others are interested and a topic that has not been reviewed recently. After identifying the topic of review, the first step is to identify broad keywords relevant to your topic and review scope. These require careful consideration, as they are responsible for directing your literature search and affect the material you will acquire to review. Later on, these keywords can be expanded to refine the search into specific subheadings and enable you to structure the main body of your review.

The next step is gathering the literature to be reviewed on a focused topic or evidence to support a hypothesis in order to contextualize research data. Thanks to the Internet that these days knowledge is at our fingertips and we can readily access online information via sophisticated search engines tools such as Google Scholar. It is essential to read published original research articles to formulate your literature review, pay attention to the research content of the articles you deem the "most relevant." Identify methodologies, the most important questions they address, if they are well-designed and executed. Try to strike a balance between old established papers and current ones, which refute as well as support a particular idea or research finding [5].

A review article should not be just about reporting published facts but it should be an informative, personal but unbiased analysis, providing a balanced view that includes inconsistencies as well as established and current developments to identify future research direction. Insightful reviews writing requires careful consideration of the published research to construct an unbiased narrative supported by published evidence. Whilst summarizing published findings, it is important for you to add perspective by commenting on the quality of the evidence presented. Interpretation of the data and assessment of the data quality are essential to give your reviews insightful and to reduce bias.

The review structure and length of in-depth analysis highly depend on the type of reviews, but in essence the major objective of all type of review articles are to provide a critical appraisal of the current knowledge on the selected research topic. Much of the work now involves extracting the critical information from the publications and organizing it in a way that highlights the issue. Finally, you must ask what sort of conclusion is appropriate. At one extreme, the conclusions are simply a condensed summary of the contents of the literature, without interpretation. Other reviews provide an overall interpretation of the literature. At the highest level, a review identifies new patterns in the data that have not been previously recognized.

While submitting review articles to Extensive Reviews journal, ask yourself whether the article represents a thoughtful and rigorous approach.

\subsection{Structuring Review Article}

Besides expertise it also requires a proper organization of the contents to represent a structured review article. The article shall include major elements of a review article such that; Title, Authors with affiliation, Abstract, Keywords, Introduction, Main Body with Suitable Headings, Discussion, Conclusion, Competing Interests, References, etc. Structure $\&$ headings of main body depends on the scope of review articles, the author shall ensure that each section of main body include suitable content specific heading and each section include author's own insight in view of objective of the article. Few additional headings may require depending on type of review article, e.g., systematic review article may also include "Review Criteria" \& "Analysis" section.

Several organizations provide guidelines for conducting an intensive literature search intended for systematic reviews by advocating on inclusion of headings such as "Methods" \& "Results", we discourage 
using of such research specific headings in review article. Instead, we encourage headings "Review Criteria" in place of Methods and Heading “Analysis" in place of Results. It's evident from past experience that many authors trying to submit review article by twisting few terms to represent it as an original research article. It might be due to the academic requirement of publishing research article however; due to lack of research they prepare review article and use research specific terms to mislead and represent the reviews as original research. The distinction in original research and reviews shall be clearly maintained and hence its more appropriate to use headings "Review Criteria" \& "Analysis" in review articles and author shall avoid using terms "this research" or "this research paper" or any other research specific terms throughout the manuscript. The authors shall also avoid using headings such as "Literature Review" or "Related Works" as the whole article is bases on literature review and hence a particular section is not required such headings.

The format of a review article can take a number of forms depending on the type of article and specific topic or subject [6]-[8] these forms will vary in methodology, rigor, and depth. Extensive Reviens journal strongly discourage terms "Methods" \& "Results" in structuring the review articles. Depending on the type of review articles, if required, author shall use headings "Review Criteria" to represent review methodology \& "Analysis" to represent result of research or data analysis from literature reviews.

\subsection{Organizing References}

While gathering \& investigating the research articles on your topic, it's critical that you keep track of all the sources. Choose the most relevant research articles and remember to keep notes of their bibliographic references to be used later on. This process will likely provide a tremendous amount of information and hence organization is crucial to use it consistently with right reference citation. Increasingly, researchers utilize citation manager tools to store references, cite, and generate bibliographies. Using of citation managers such as Endnote, Evernote, RefWorks, Papers, Zotero, Mendeley, etc. can be helpful as they keep track of and cite sources as you go through the research process however, all such tools are not free to use.

Here, I would like to mention 2 free and user-friendly tools that I frequently use as citation manager, i.e., Mendeley [9] \& Zotero [10]. There is another online free tool by Zotero to create a quick bibliography called ZoteroBib [11] for those who is not comfortable in using citation manager but would like to format the reference list in a standard and consistent style. The author shall carefully cite only most relevant references and avoid bunch of in-text citation within a single sentence. The citation shall be discussed and reflect the accuracy for the claimed content.

Extensive Reviens journal allows a maximum. of 25 citations/1000 words with a limit of max 300 references for whole review articles out of which $50 \%$ references shall be recent from the last 2 years. Further, to avoid possible bias self-citation is limited to less than $20 \%$ of total citations. Here self-citation means citation of articles by same author, a common author, from same laboratory, or from the same journal. The preferred citation style for this journal is IEEE style.

\section{$5 \quad$ How Should the Review Articles be Used?}

Reviews provide a quick way to gain some familiarity with the issues and major contributors. They may also provide useful syntheses and critical insights. However, their conclusions must be used with caution. Reviews should not be used as a substitute for reading the original research papers that are important to your own work. It is particularly bad practice to cite a paper based on the description of it in a review. It's because reviews depend on a certain approach of author in terms of how published work is reviewed and discussed. A lack of critical appraisal or systematic approach can propagate erroneous conclusions. By all means, take advantage of reviews; but do not consider them to be the final authority.

As fresh research data and new innovations are introduced, the need for updating scholarly reviews on a particular topic always required. In updating the review article, the authors convey to readers that what 
recent knowledge and ideas has been established on the topic, what their strengths and what is the weaknesses which still needs to be address.

\section{Reflections on the Impact of a Review Article}

The continuously growing number of research publications can be overwhelming to stay updated with recent developments on a particular topic. A review article provides an overview and an evaluation of the current state of knowledge about a specific area of research. It may also include a discussion of methodological issues and suggestions for future research. In this way the review articles considered as a powerful tool to provide an updated research development that allows easy access to a research field, especially for young researchers and graduate students or experienced researchers; looking for further research direction. Therefore, authoring review articles bears a great responsibility in terms of how published work is reviewed and discussed. A lack of critical appraisal or systematic approach can propagate erroneous conclusions. However, well-synthesized reviews often guide the future studies and might thus influence the research in the field.

Writing a review article requires to follow a specific structure, e.g., it is expected that you have an introduction - that provides a general idea of the focused research topic and organizational pattern of the article; A body - that contains focused sections having suitable headings for the actual discussion of the research data with author's own insight; and finally the conclusions- which represent your conclusive remarks and recommendations that have been drawn from the reviewed research work. The greatest impact of a review comes through a meta-analysis that executes detailed statistical analysis systematically from independent research papers. Outcomes from meta-analyses often have the highest strength of evidence but it must be noted that a meta-analysis is only as good as the papers it includes. If their information in selected research work is flawed, the meta-analysis may be statistically strong but invalid.

Authors tend to cite a review article more often compared to original research work that raise concerns for few authors as it lowering the number of citations for the respective articles. However, on the other hand, if studies are included and discussed in a review with proper citation, scholars who would like to learn more or access the original perspectives tend to access the original research work and possibly cite them as well. The benefits of publishing review articles clearly outweigh any potential shortcomings. A review article must fulfil these things:

- be organized around and related directly to the research question you are developing.

- synthesize the review with what is known and what is not known in an organized manner.

- identify areas of controversy in the research work.

- justify the need of further research.

Extensive Reviews journal is inviting experts to submit insightful review articles on high-priority trending research topics.

\section{Conclusions}

The insightful review article writing is a vital part of research and should occur through the extensive review process to help researchers designing a strong study, effectively communicating results and representing its importance. To achieve these goals, researchers are advised to plan and execute the literature review carefully. This editorial provides importance of review article along with considerations and recommendations that may improve the quality of reviews article preparation as per expectation of the Extensive Reviews Journal. In short-

- A review article should demonstrate current knowledge, identify gaps in the field and, if relevant, demonstrate what shall be future research direction.

- It should be a personal critical appraisal of the latest development in a particular research topic.

- It should be based on the critical reviews of variety of original research articles \& reporting facts.

- Structure your review with an introduction, Main Body and Conclusions. 
- Summaries research information in your own words and give appropriate credit to other authors' work by appropriate citation.

- A systematic approach to writing a review article can be used to reduce bias.

\section{Competing Interests}

The author is an editorial board member of this journal.

\section{How to Cite this Article:}

A. A. Bahishti, "The Importance of Review Articles \& its Prospects in Scholarly Literature”, Extsv. Rev., vol. 1, no. 1, pp. 1-6, Sep. 2021. https://doi.org/10.21467/exr.1.1.4293

\section{References}

[1] M. Dutta, "The Importance of Scholarly Reviews in Medical Literature," Ear, Nose \& Throat Journal, vol. 98, no. 5, pp. 251-252, Jun. 2019. https://doi.org/10.1177/0145561319827725.

[2] G. W. Suter, "Review papers are important and worth writing," Environ. Toxicol. Chem., vol. 32, no. 9, pp. 1929-1930, Sep. 2013. https://doi.org/10.1002/ETC.2316.

[3] A. A. Bahishti, "Peer Review: Critical Process of a Scholarly Publication,” J. Mod. Mater., vol. 2, no. 1, pp. 1.1-1.2, Oct. 2016. https://doi.org/10.21467/jmm.2.1.1.1-1.2.

[4] A. Bahishti, "Simultaneous Submission of Journal Article: A Serious Author Misconduct," AIJR Preprints, 22, Sep. 2020. https://doi.org/10.21467/PREPRINTS.22.

[5] L. Winchester and M. Salji, "Writing a literature review”, Journal of Clinical Urology, vol. 9, no. 5, pp. 308-312, May 2016. https://doi.org/10.1177/2051415816650133.

[6] Nicole Graulich, S. E. Lewis, Ajda Kahveci, J. M. Nyachwaya, and G. A. Lawrie, "Writing a review article: what to do with my literature review," Chem. Educ. Res. Pract, vol. 22, no. 3, pp. 561-564, Jul. 2021. https://doi.org/10.1039/D1RP90006D.

[7] S. Bacq, W. Drover, and P. H. Kim, "Writing bold, broad, and rigorous review articles in entrepreneurship," J. Bus. Ventur., vol. 36, no. 6, p. 106147, Nov. 2021. https://doi.org/10.1016/J.JBUSVENT.2021.106147.

[8] W. R. Clark, L. A. Clark, D. M. Raffo, and R. I. Williams, "Extending Fisch and Block's (2018) tips for a systematic review in management and business literature," Manag. Rev. Q. 2020 711, vol. 71, no. 1, pp. 215-231, Mar. 2020. https://doi.org/10.1007/S11301-020-00184-8.

[9] Mendeley, "Reference Manager and Academic Social Network - Mendeley Database | Elsevier Solutions." https://www.elsevier.com/solutions/mendeley (accessed Sep. 12, 2021).

[10] "Zotero | Your personal research assistant." https://www.zotero.org/ (accessed Sep. 12, 2021).

[11] "ZoteroBib: Fast, free bibliography generator - MLA, APA, Chicago, Harvard citations." https://zbib.org/ (accessed Sep. 12, 2021).

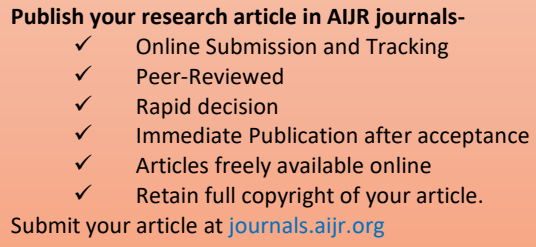

Publish your books with AIJR publisherPublish with ISBN and DOI. Publish Thesis/Dissertation as Monograph. Publish Book Monograph. Publish Edited Volume/ Book. Publish Conference Proceedings Retain full copyright of your books. Submit your manuscript at books.aijr.org 